\title{
Atriopulmonary connection for mechanically assisted Fontan completion: Classic technique for modern strategy
}

Iki Adachi, MD, ${ }^{\mathrm{a}}$ Hari Tunuguntla, MD, ${ }^{\mathrm{b}}$ Barb Elias, RN, ${ }^{\mathrm{a}}$ Prakash M. Masand, MBBS, MD, ${ }^{\mathrm{c}}$ and Sebastian C. Tume, MD, ${ }^{\mathrm{d}}$ Houston, Tex

\footnotetext{
From ${ }^{a}$ Congenital Heart Surgery, Department of Surgery, ${ }^{\mathrm{b}}$ Pediatric Cardiology, ${ }^{\mathrm{c}}$ Pediatric Radiology, and ${ }^{\mathrm{d}}$ Pediatric Critical Care, Department of Pediatrics, Texas Children's Hospital and Baylor College of Medicine, Houston, Tex.

Disclosures: Dr Adachi serves as a consultant/proctor for Berlin Heart, Medtronic, Jarvik, BiVACOR, and Sony-Olympus Medical Solutions. All other authors reported no conflicts of interest.

The Journal policy requires editors and reviewers to disclose conflicts of interest and to decline handling or reviewing manuscripts for which they may have a conflict of interest. The editors and reviewers of this article have no conflicts of interest.

Received for publication May 15, 2020; revisions received May 15, 2020; accepted for publication June 17, 2020; available ahead of print June 21, 2020.

Address for reprints: Iki Adachi, MD, Mechanical Circulatory Support, Congenital Heart Surgery, Texas Children's Hospital, Michael E. DeBakey Department of Surgery, Baylor College of Medicine, 6651 Main St, Houston, TX 77030 (E-mail: iadachi@bcm.edu).

JTCVS Techniques 2020;3:307-9

2666-2507

Copyright (C) 2020 The Authors. Published by Elsevier Inc. on behalf of The American Association for Thoracic Surgery. This is an open access article under the CC BY-NCND license (http://creativecommons.org/licenses/by-nc-nd/4.0/).

https://doi.org/10.1016/j.xjtc.2020.06.019
}

$\rightarrow$ Video clip is available online.

With the evolution of single-ventricle palliations, "classic Fontan" with atriopulmonary connection (APC) has been abandoned due to unfavorable long-term sequelae. ${ }^{1}$ However, these negative sequelae are avoidable if the duration of Fontan circulation is limited to the wait for heart transplantation. Recently, Fontan operation with concomitant placement of a ventricular assist device (VAD)mechanically-assisted Fontan completion ${ }^{2}$ - has emerged as a promising strategy for failing Glenn circulation. ${ }^{3,4}$ Here we describe our experience of the APC Fontan with VAD placement. The patients' legal guardians provided informed consent for the publication of the images and video presented in this report.

\section{CASE DESCRIPTION}

An inotropic-dependent 2-year-old male $(12.6 \mathrm{~kg}$, body surface area, $0.58 \mathrm{~m}^{2}$ ) with failing Glenn physiology was transferred to our intensive care unit. Cardiac anatomy was right ventricle (RV)-dominant atrioventricular (AV) septal defect and double-outlet $\mathrm{RV}$ with right atrial isomerism. At a neighboring institution, he had undergone pulmonary artery (PA) banding as a neonate, followed by

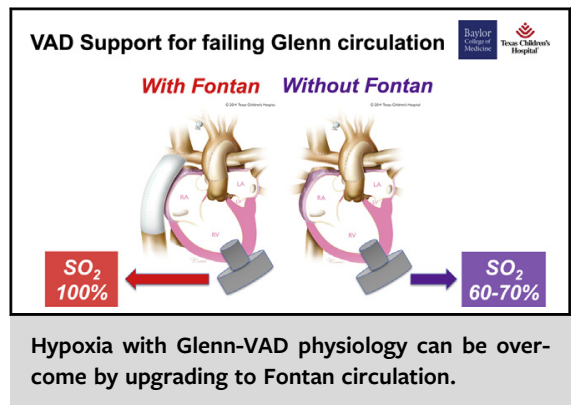

CENTRAL MESSAGE

Fontan completion with VAD

placement has emerged as a

promising strategy for failing

Glenn circulation. "Classic Fon-

tan" with an atriopulmonary

connection is an alternative to

this modern strategy.

See Commentary on page 310.

bilateral bidirectional Glenn shunt with AV valve repair at 9 months. An attempted AV valve replacement resulted in progression of heart failure and transfer of care to our facility.

A computed tomography scan showed severe compression of the left pulmonary veins associated with diminished left lung volume due to massive cardiomegaly (Figures 1 and 2). Cardiac catheterization demonstrated preserved pulmonary vascular resistance $\left(2.4 \mathrm{WUm}^{2}\right)$ and substantially elevated Glenn and PA capillary wedge pressures (30 and $26 \mathrm{~mm} \mathrm{Hg}$, respectively). Given the patient's clinical deterioration on inotropes (INTERMACS profile 2), the need for VAD support became unavoidable.

Via repeat median sternotomy, cardiopulmonary bypass was established. Under deep hypothermic circulatory arrest, the left-sided inferior vena cava (IVC) was baffled to the right-sided atrium with a bovine pericardial patch with a 6-mm fenestration, such that the majority of the rightsided atrial cavity would serve as a Fontan channel. The roof of the right-sided atrium was anastomosed to the PA (Figure 1). The Heartware VAD (HVAD; Medtronic, 


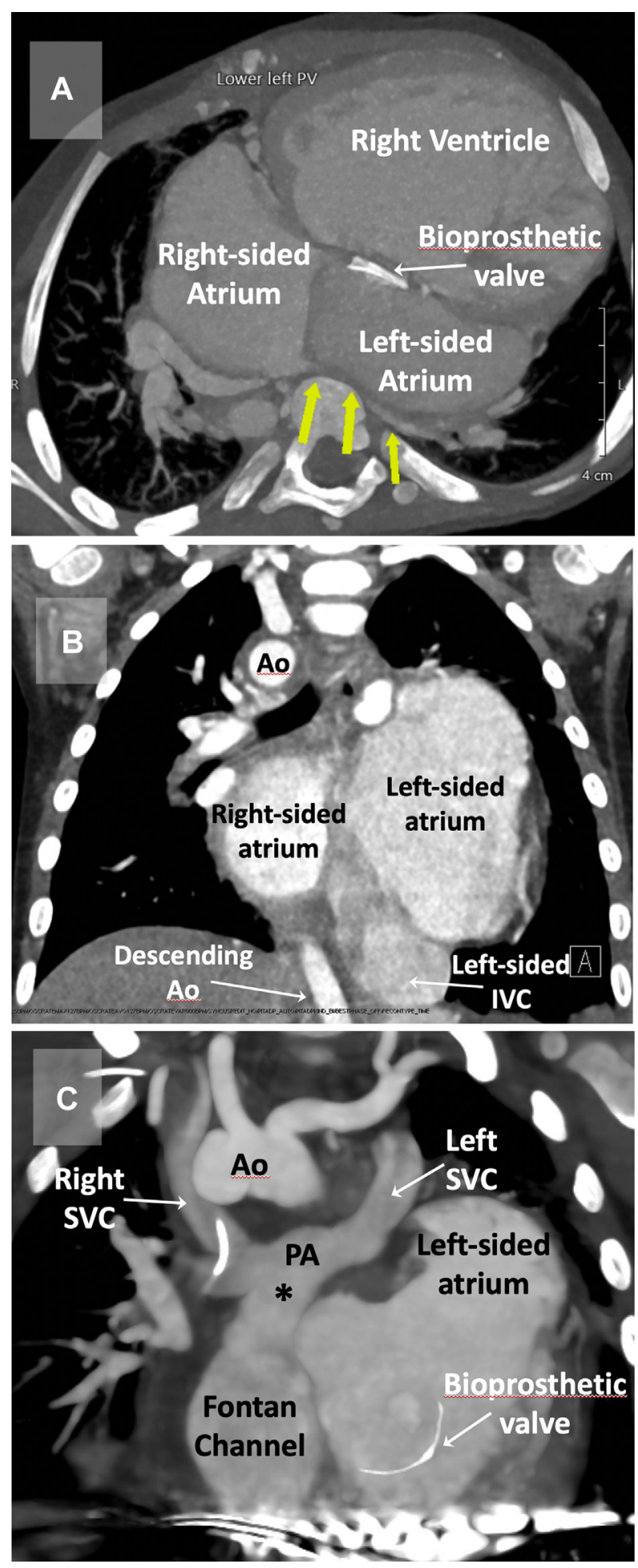

FIGURE 1. Preoperative and postoperative computed tomography scans. A and B, Preoperative images show near-complete occlusion of the left pulmonary vein (yellow arrows) owing to compression from the dilated atrium and the spine. The inferior vena cava is to the left of the spine. C, Postoperatively, the right-sided atrium serving as a Fontan channel is connected to the central pulmonary artery in an unobstructed fashion (marked with an asterisk). PV, Pulmonary vein; Ao, aorta; $I V C$, inferior vena cava; $P A$, pulmonary artery; $S V C$, superior vena cava.
Minneapolis, Minn) was inserted in the diaphragmatic surface of the RV. The HVAD outflow graft was anastomosed to the ascending aorta. After HVAD activation, the patient was weaned from bypass.

The patient was extubated on postoperative day 2 and weaned off inotropes the next day. Bivalirudin was used to transition to coumadin with aspirin. He developed a small right subarachnoid hemorrhage and a left subdural hemorrhage at 3 weeks after surgery, necessitating reversal of anticoagulation for 1 week. A postoperative computed tomography scan showed widely patent Fontan channel (Figure 1). Cardiac catheterization demonstrated improved hemodynamics (Fontan and PA capillary wedge pressure 12 and $8 \mathrm{~mm} \mathrm{Hg}$, respectively), with a systemic oxygen saturation of $85 \%$. Just before home discharge (at 9 weeks postoperatively), pump thrombosis developed, necessitating HVAD exchange via sternotomy on bypass. He was extubated the same day and subsequently recovered without sequelae. A suitable cardiac organ became available 1 week later. Bilateral Glenn anastomoses were taken down and reconstructed with the donor superior vena cava (SVC) and the innominate vein. The leftward IVC with the atrial cuff was anastomosed to the donor IVC. The patient was extubated on postoperative day 3 and was weaned off inotropes 2 days later. Following uneventful recovery, the patient was discharged home on postoperative day 23 .

\section{DISCUSSION}

VAD support for failing Glenn circulation remains a challenge, with 6 -month survival of $20 \%$ to $40 \%$. $^{3,4}$ Among multiple factors for suboptimal outcomes, dichotomous systemic venous return (ie, pressure differential between the SVC and IVC) warrants attention. By decompressing the systemic ventricle with VAD (thereby the IVC system only), the pressure differential will be further aggravated, resulting in worsening cyanosis $^{5}$ (Figure 3 and Video 1). Therefore, the benefit of improved cardiac output with VAD is negatively offset by worsening hypoxia, which jeopardizes the fundamental goal of VAD support (improved oxygen delivery). To tackle this Glenn-specific issue, 2 approaches have been proposed: Glenn takedown (downgrade from stage 2 to stage 1$)^{4}$ and complete Fontan (upgrade to stage 3 ). ${ }^{2}$ Given the VAD survival difference ( $\approx 30 \%$ for stage 1 and $\approx 95 \%$ for stage 3 at 6 months), ${ }^{3}$ the latter may be more appealing.

If the goal of Fontan completion is to improve the efficacy of VAD support, then APC would be a reasonable alternative to the standard total cavopulmonary connection. The disadvantages of APC stem primarily from its long-term sequelae. Thus, if the expected duration of Fontan circulation is limited (ie, bridge to 


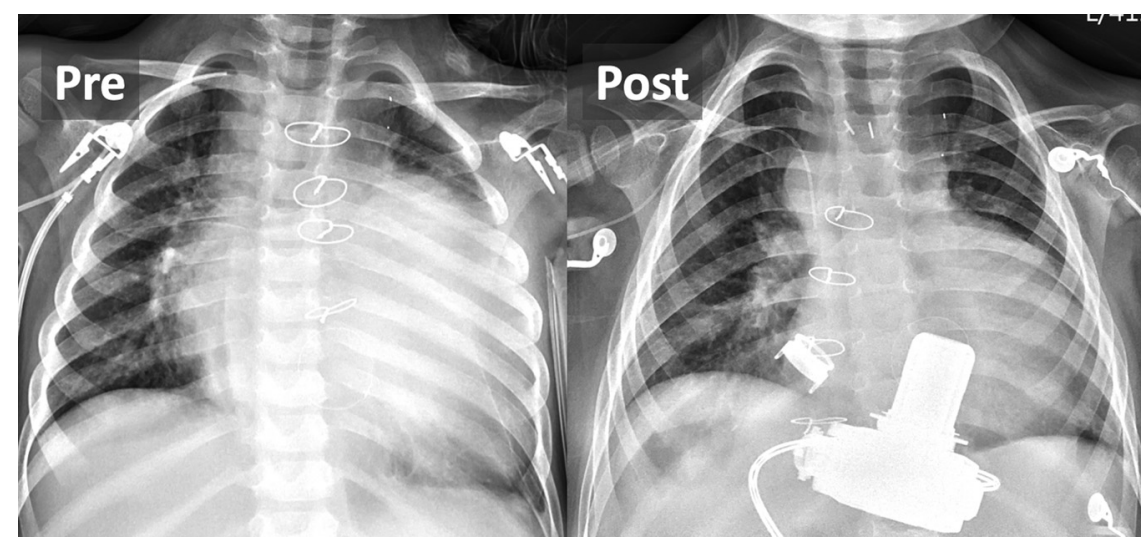

FIGURE 2. Preoperative and postoperative chest X-rays showing a marked reduction in the size of the heart.

transplantation), the technical advantage of the classic procedure would outweigh its long-term drawbacks. The APC would be a valuable option in patients with complex systemic venous return. The beauty of the APC comes to fruition at VAD explantation/transplantation; the large area of the atrial free wall remains available for cannulation, which simplifies bypass initiation. In our case, cardiopulmonary bypass was established with a single atrial cannula before transitioning to the tricaval cannulation; the leftward IVC was not accessible until the HVAD was explanted.

We are deeply grateful to Professor Fontan, who provided generous grant support and intellectual guidance through the Fontan Prize of the European Association for Cardio-Thoracic Surgery. We also express our sincere gratitude to Dr John Lamberti (Stanford University) for providing the inspiration for a new role of the classic Fontan in the current era.

\section{VAD Support for failing Glenn circulation}
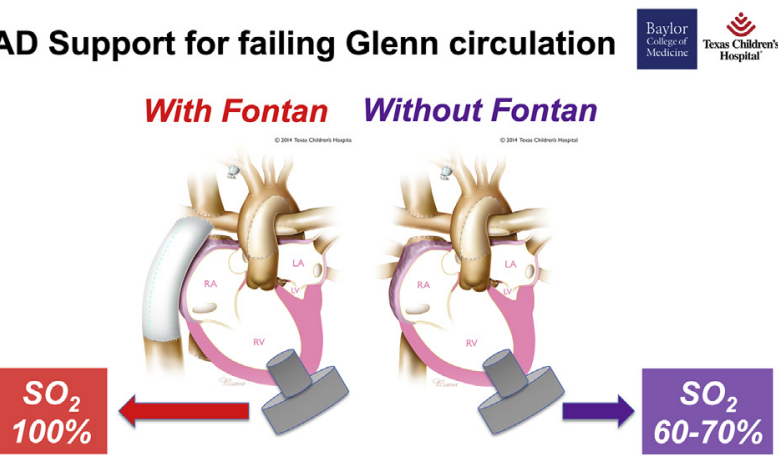

FIGURE 3. Difference in systemic saturation with or without Fontan upgrade. Owing to the dichotomous systemic venous flow issues unique to the Glenn circulation, profound hypoxia can occur in patients with VAD support without conversion to the Fontan circulation. $V A D$, Ventricular assist device; $L A$, left atrium; $R A$, right atrium; $L V$, left ventricle; $R V$, right ventricle.

\section{References}

1. Fontan F, Baudet E. Surgical repair of tricuspid atresia. Thorax. 1971;26:240-8.

2. Adachi I, Williams E, Jeewa A, Elias B, McKenzie ED. Mechanically assisted Fontan completion: a new approach for the failing Glenn circulation due to isolated ventricular dysfunction. J Heart Lung Transplant. 2016;35:1380-1.

3. Peng DM, Koehl DA, Cantor RS, McMillan KN, Barnes AP, McConnell PI, et al Outcomes of children with congenital heart disease implanted with ventricular assist devices: an analysis of the pediatric interagency registry for mechanical circulatory support (Pedimacs). J Heart Lung Transplant. 2019;38:420-30.

4. Chen S, Rosenthal DN, Murray J, Dykes JC, Almond CS, Yarlagadda VV, et al Bridge to transplant with ventricular assist device support in pediatric patients with single ventricle heart disease. ASAIO J. 2020;66:205-11.

5. Adachi I. Ventricular assist device support for complex congenital heart disease: inspiration from history of surgical evolution. J Heart Lung Transplant. 2019; $38: 431-2$.

6. Adachi I, Jeewa A, Burki S, McKenzie ED, Fraser CD Jr. Outpatient managemen of a child with bidirectional Glenn shunts supported with implantable continuousflow ventricular assist device. J Heart Lung Transplant. 2016;35:688-90.

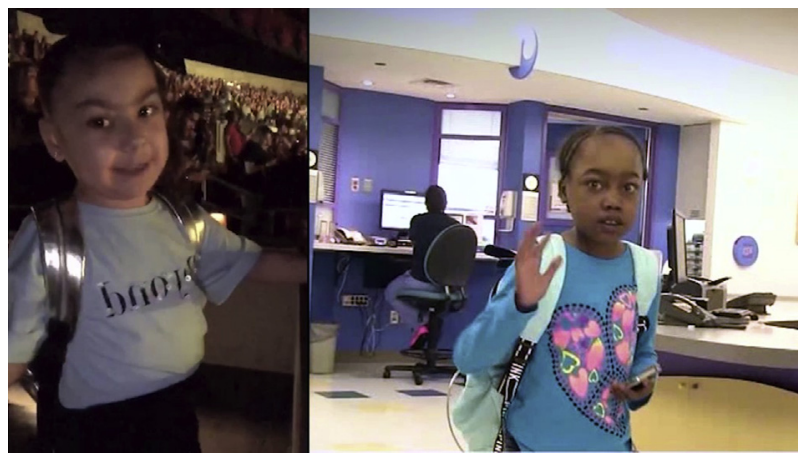

VIDEO 1. Short video clips showing the difference in exercise capacity in patients receiving HeartWare ventricular assist device (HVAD) support for failing Glenn circulation with Fontan upgrade (left; a 6-year-old with a history of hypoplastic left heart syndrome) or without Fontan upgrade (right; a 10-year-old with heterotaxy syndrome: the detail reported by Adachi and colleagues $^{6}$ ). Both clips were recorded approximately 2 months after the HVAD placement. The patients' legal guardians provided informed consent for the publication of the study data, including the use of images and video. Video available at: https://www.jtcvs.org/article/S26662507(20)30297-2/fulltext. 